\title{
The Presence or Absence of Melanocytes in the Human Optic Nerve and Its Meningeal Sheaths
}

\author{
KASEI KOJIMA, ARATA SUGITA AND HISAHARU YOSHIOKA
}

Department of Ophthalmology, Kurume University School of Medicine, Kurume, 830 Japan

Received for publication April 25, 1984

\begin{abstract}
Key words: human eye - optic nerve - optic nerve sheaths - melanocytes light microscopy
\end{abstract}

Vogt-Koyanagi-Harada disease has been recognized to be an autoimmune disorder with hypersensitivity to melanocytes as the possible autoantigen. The state of melanocytes distribution has been thought to be closely related to the clinical features of the disease.

Clinically, the optic neuritis has been rarely present in cases of Vogt-KoyanagiHarada disease reported in Japan (Yamaguchi, 1954: Yoshioka et al. 1983) and is prone to be regarded as questionable in association with the disease. This contrasts sharply with reports from other countries showing the presence of this complication in $35 \%$ of cases (Ohno et al. 1977).

As yet, to the best of our knowledge, there has been no article dealing with a detailed histologic observation of the optic nerve and its meningeal sheaths for occurrence and distribution of melanin-containing cells. It constitutes a clinically impor tant problem whether melanocytes are distributed in the human meningeal sheaths of the optic nerve or not in exploring the the possibility of the development of optic neuritis in Vogt-Koyanagi-Harada disease.

In the present study, we tried to ascertain whether or not melanocytes might exist and to investigate their distribution histologically in the optic nerve and its meningeal sheaths of human eyes.

This report documents the findings noted in 43 eyes from 29 cadavers. To obtain cadaveric eyes including the optic nerve, the paries superior of the base of the skull was destroyed after removal of the skullcap and brain, followed by orbital periosteomy. The optic nerve was then resected from the eyeball together with the ocular tissue surrounding the optic nerve entrance to the bulbus, encompassing the optic disc, retina, choroid and sclera. Each optic nerve was cut at the entrance and outlet of the optic canal and, centrally, through a sagittal palne of the chiasma. Microscopic observations were made of the intraocular and orbital portions of the optic nerve in this study.

These optic nerves were fixed in Bouin solution and embedded in paraffin and then were dried for more than 2 hours in a dessicator. Then materials were dehydrated in xylene and through a graded series of alcoholes. Meridional serial sections $(4 \mu \mathrm{m})$ were cut with an Americal Optical 820 Spencer microtome. These sections were stained with hematoxylin and eosin and observed with a light microscope.

In two $(4.7 \%)$ of forty three eyes, melanocytes were observed in the meningeal sheaths of the optic nerve. They were seen aggregated in the pia or dura just central to the lamina scleralis (Fig. 1, $2,3)$. There was no microscopic evidence of melanocytes within the optic nerve and 
lamina cribrosa in any of the cases studied.

It is widely recognized that melaninbearing cells exist in the pia mater of meninx (Bloom and Fawcett, 1975) and that the presence of melanocytes has a pathogenetic relation in the development of meningitis which occurs in association with Vogt-Koyanagi-Harada disease.

The presence of melanocytes in the optic nerve sheaths continuous with the meninx has been described in cases with melanocytosis of the optic disc (Reese, 1976) but it was quite unknown whether melanocytes occur in normal optic nerves or their meningeal sheaths of the human eye.

The present observation of melanocytes present in the pia and dura mater of the optic nerve sheaths, being first to be reported in the literature, would strongly suggest a possible, rare involvement of optic neuritis secondary to piamatral and duramatral inflammation in Vogt-Koyanagi Harada disease in Japan.

Acknowledgment: The authors are indebted to Professor Michio Miyazaki and Yasuhiro Katsume M. D. of the Department of Anatomy, Kurume University School of Medicine for their cooperation. Technical work was done by Miss Emiko Hiromatsu.

\section{References}

YAMAGUCHI, M. (1954). Two cases of Harada's Disease with a picture of acute retrobulbar neuritis at the outset. Rinsho Ganka (Jpn. J. Clin. Ophthalmol. ), 8, 715-718.

Yoshioka, H., Sugita, T. and Nagahama, M. (1983). Optic neuritis, sub-pigment epithelial hemorrhage or Elschnig's spot-like lesion associated with Vogt-Koyanagi-Harada disease. Rinsho Ganka (Jpn. J. Clin. Ophthalmol.), 37, 467-475.

OhNo, S., Char, D. H., Kimura, S. J. and O'Connor, G. R. (1977). Vogt-Koyanagi-Harada syndrome. Am. J. Ophthalmol. 83, 735-740.

Bloom, W. and Fawcett, D. W. (1975). A textbook of histology. Tenth edition, pp. 377. Philadelphia: W.B. Saunders Co.

Reese, A. B. (1976). Tumors of the Eye. Third edition. pp. 187-188. New York: Harper and Row, Publishers. 


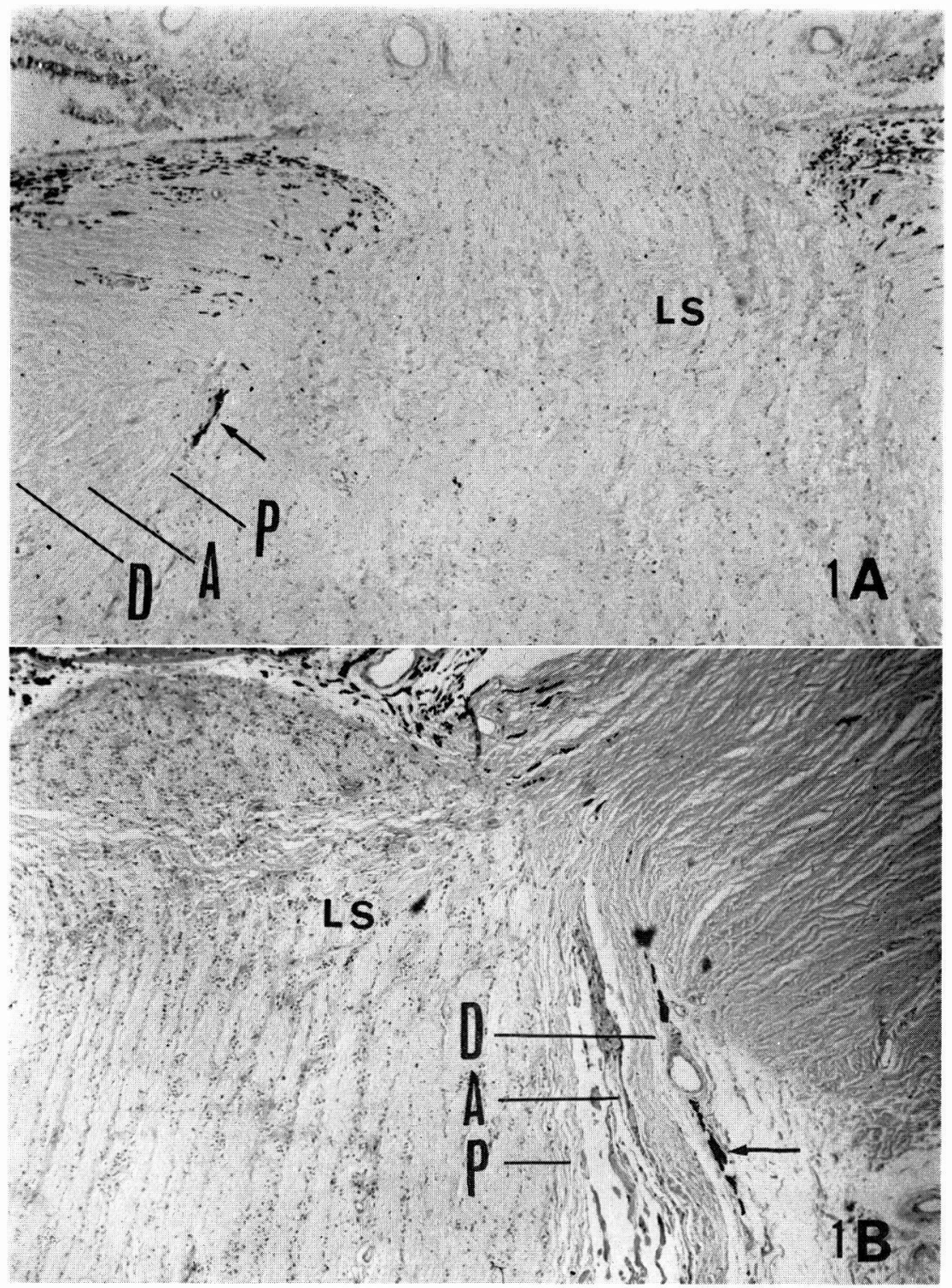

Fig. 1. A photomicrograph of melanocytes (arrow) in the pia just central portion of the lamina scleralis in cadaver No. 19 (1 A) and in the dura just central portion of the lamina scleralis in cadaver No. 2 (1B). P: pia mater, A: arachnoid mater, D: dura mater, LS: lamina scleralis. $\times 16$ 


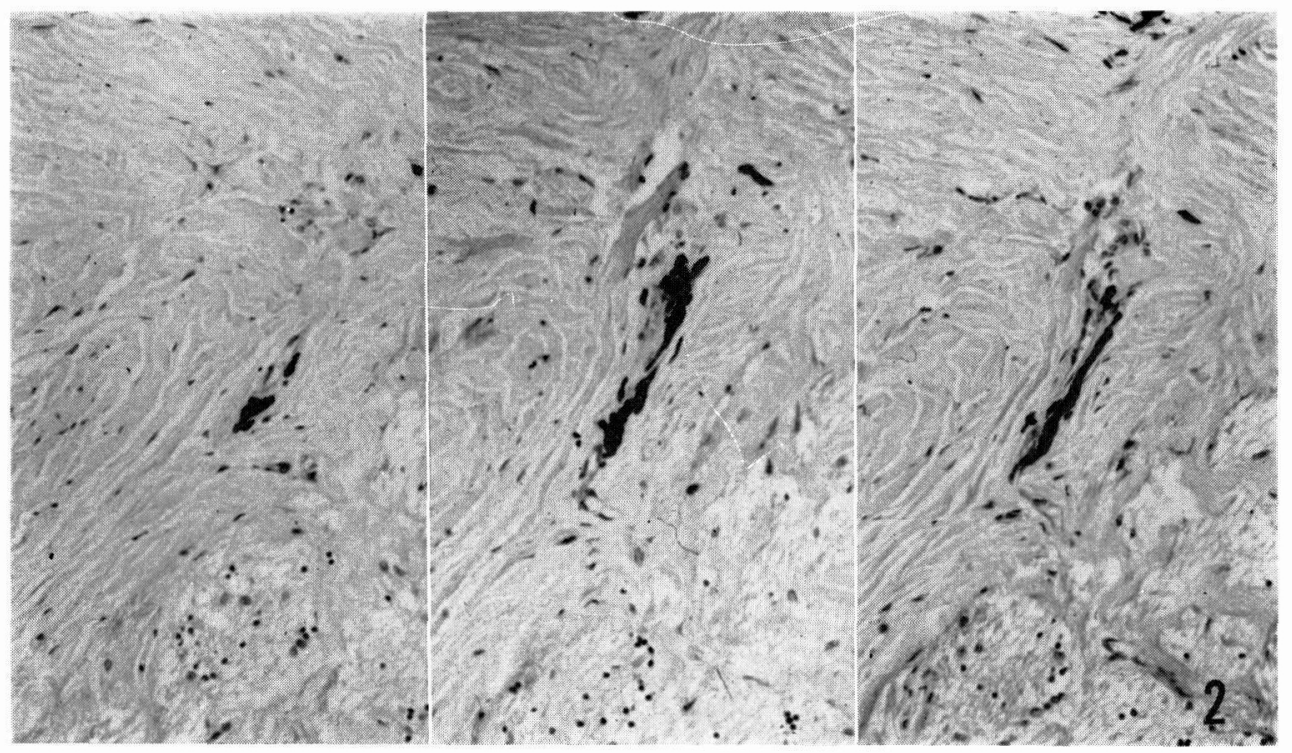

Fig. 2. serial section of the melanocytes in Fig. 1 A. $\times 40$

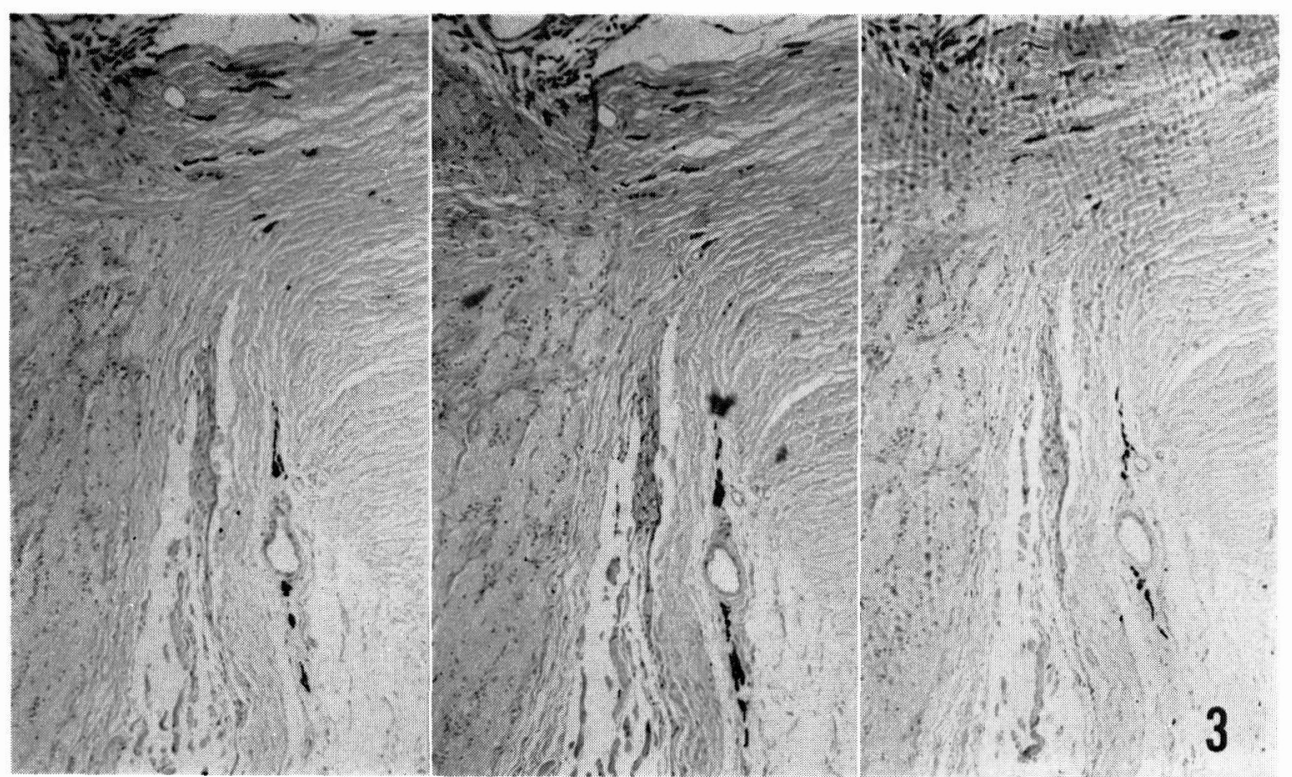

Fig. 3. serial section of the melanocytes in Fig. 1B. $\times 16$ 